\title{
Improved grid integration of intermittent electricity generation using electric vehicles for storage: A simulation study
}

\author{
Paul Monigatti
}

paulmoni@waikato.ac.nz

\author{
Mark Apperley \\ University of Waikato \\ Hamilton, New Zealand \\ m.apperley@cs.waikato.ac.nz
}

Bill Rogers

coms0108@cs.waikato.ac.nz

\begin{abstract}
This paper describes a simulation to establish the extent to which reliance on non-dispatchable energy sources, particularly wind generation, could in the future be extended beyond accepted norms, by utilizing the distributed battery capacity of an electric vehicle fleet for storage. The notion of exploiting the distributed battery capacity of an electric vehicle fleet as grid storage is not new. However, this simulation study specifically examines the potential impact of the idea in the New Zealand context. The simulation makes use of real and projected data in relation to vehicle usage, full potential wind generation capacity and availability, taking into account weather variation, and typical daily and seasonal patterns of electricity usage. It differs from previous studies in that it is based on individual vehicles, rather than a bulk battery model. At this stage, the simulation does not take into account local or regional flows. A more detailed analysis of these localized effects will follow in subsequent stages of the simulation work.
\end{abstract}

Keywords-electric vehicles, wind energy, smart grids, V2G, simulation

\section{INTRODUCTION}

The notion of utilizing the aggregated battery capacity of an electric vehicle (EV) fleet as storage on the electricity grid (V2G) is not new. Fifteen years ago, Kempton and Letendre [1] reported a detailed analysis of the potential benefits of such a scheme, in both energy and economic terms. The basic concept is that, as electrically powered road vehicles become more common, their need to be regularly connected to the electricity grid in order to have their batteries charged can be exploited by regarding them as both a load and a source. By ensuring that vehicles are connected to the grid ("plugged-in") whenever possible (i.e. when not being driven), and by arranging for the immediate future requirements of each car to be specified (next journey time, next journey distance), surplus electricity can be stored in the batteries. At times when demand exceeds the available generation capacity, energy can be drawn from the batteries, provided each individual vehicle has sufficient charge remaining at the stated time for its next departure to complete that journey.

Heightened interest in better integrating renewable and non-dispatchable energy sources (typically wind, but may include other technologies) into electricity grids has provided a strong motivation to explore and develop this concept. It is fundamental to the increased penetration of wind generation systems into electricity grids that, because of nondeterministic fluctuations (intermittency) of output [2], either additional back-up capacity or some form of storage is required. This is especially important as penetration grows to more than $\sim 20 \%$ of the total supply [3], [4]. The provision of back-up capacity constitutes an inefficiency in overall grid system design [5], [6], leading to a focus of interest in range of potential storage technologies [5], [7-9]. Peak shifting and smoothing can act as virtual storage, and reduce the total storage requirement [10]. Others have shown that with appropriate and adequate storage, wind energy penetration can be increased to as much as $65 \%$ of total generation capacity [9]. The serendipitous match between the need to reduce fossil fuel dependency (and $\mathrm{CO}_{2}$ emissions) in both vehicle fleets and electricity generation, and the consequent growth in both battery capacity and dependency on nondispatchable renewable energy sources, has not gone unnoticed [2], [4], [10-14]. Coupled with the fact that overall vehicle utilization is reckoned to be as low as 4\% [4], the notion of exploiting the under-utilized electrical storage capacity of a national fleet of electric vehicles to balance the fluctuations inherent in new forms of electricity generation has many attractions.

Earlier research on this topic, generally characterized as vehicle-to-grid, or V2G, technology, has explored economic and business models [5], [11], [15], [16], as well as vehicle technologies and connection management [4], [11], and overall feasibility and system impact [3], [17].

This paper describes a simulation model developed to explore the energy balance characteristics of $\mathrm{V} 2 \mathrm{G}$ in the New Zealand energy environment. New Zealand's electricity generation is primarily hydroelectric, with an increasing proportion of wind. The next section provides details of the generation mix.

The simulation incorporates accurate wind speed data, and utilizes actual corresponding grid load over a one-year period. It differs from earlier studies (for example, [10], [16]) in that it incorporates a discrete vehicle behavior and battery model, based on known statistical usage patterns and representative technologies, rather than an aggregated battery. In other studies, Ma et al. [13] have used a discrete battery model, but do not model vehicle behavior, while Waraich et al. [14] have used both a discrete battery model and modeled vehicle behavior, but have not investigated electricity supply variations or V2G aspects.

It is the energy balance capability that is being explored, specifically in seeking means by which non-dispatchable renewables can be more effectively integrated into the grid, and it is acknowledged that there are other important aspects of V2G implementation that are not covered by the present study. These include economic and business models, and the 
personal and social implications of large-scale adoption of such a scheme. Specific approaches to battery recharging provision (e.g. plug-and-charge, battery swap, inductive charging) are not addressed here, nor does the model yet take account of fine details of battery charging regimes or the charge cycle impact on battery life. However, the use of a discrete vehicle model does allow the simulation to include any combination of vehicle technologies. For example, a mix of straight plug-and-charge (BEV), smart charge (SEV) and energy-storage (V2G) vehicles can be readily included in a simulation run.

To the best of the authors' knowledge, this simulation is the first of its type to run over a complete simulated year, using real wind speed and electricity consumption data, with a discrete vehicle and battery model. Similar work typically focusses on short-term aspects over a typical day [12-14], or up to one week [16]. The long-term approach takes into account seasonal variations in both electricity load and wind generation. This capability is especially important in New Zealand, where long-term energy security during dry years is an ongoing concern due to the high dependency on hydroelectric generation.

\section{REQUIREMENTS OF THE SIMULATION}

The simulation attempts to model the potential impact of utilizing the distributed battery capacity of an electric vehicle fleet to allow more efficient and effective integration of intermittent renewable generation capacity, specifically wind, in New Zealand. The main aim of the study is to determine the extent to which load balance can be maintained with an increased proportion of wind generation capacity, and without the need for a corresponding increase in standing and spinning reserves. To provide an effective evaluation of this scenario, the simulation must accurately model the present situation in terms of electricity generation and demand, and the behavior of New Zealand's vehicle fleet: how many trips are made; at what time of day; how long those trips are and average speed of those trips. It must also accurately model wind fluctuation, and in order to incorporate seasonal variation, should be capable of working with a whole year's data. In addition, the broad specifications of present day electric vehicles are relevant as they dictate the available battery capacity and charging requirements.

Another requirement is modular design; this paper does not include intermittent generation sources other than wind, nor does it explore different demand and dispatch strategies. Careful attention to modularity in the design will enable the inclusion of these features in future work to be straightforward.

\section{A. Electricity in New Zealand}

New Zealand's electricity network has $9.4 \mathrm{GW}$ of generation capacity, generating 43 TWh annually [18], illustrated by source in Fig. 1. In 2010, renewable sources generated $74 \%$ of this energy; a figure that the government wishes to increase to $90 \%$ by 2025 as long as security of supply can be maintained [19].

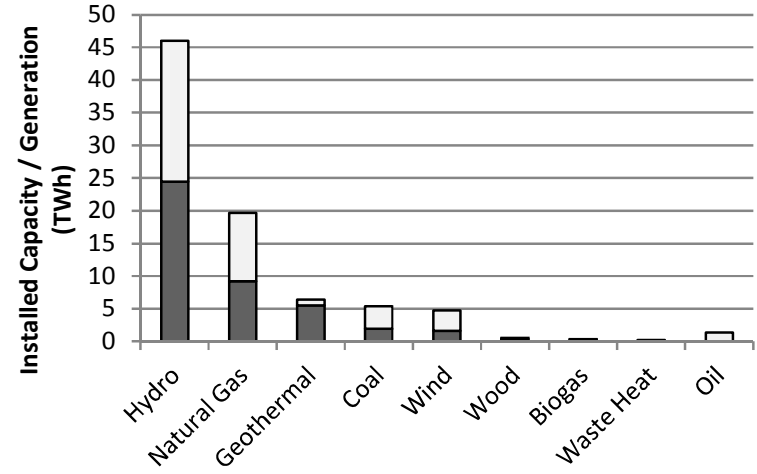

Figure 1. New Zealand Generation and Installed Capacity by source [18].

Wind generation has become of increasing interest, as New Zealand's average capacity factor for wind farms is $40 \%$, in contrast to a global average of only $22 \%$ [20].

It is important to note that hydroelectric generation is an intermittent energy source, but on a much longer time-scale than wind. The storage inherent in hydro lakes allows hydroelectric generators to act as a dispatchable source in the short to medium term.

Because of the high dependency on hydroelectric generation, New Zealand suffers electricity shortages during dry years. Bardsley [21] has proposed long-term pumped hydro to help mitigate these effects, while Bull [22] has shown a correlation between low hydro inflows and low average wind speed, implying that dry years may also be calm years, and recommends reducing the assumed contribution of wind generation by $10 \%$ [22]. Although the simulation is capable of exploring these issues, they have been left to future work.

\section{B. Vehicle Fleet in New Zealand}

New Zealand is a small country with a population of approximately 4.4 million [23], and in December 2010, had 2,599,568 light passenger vehicles [24]. These light passenger vehicles make up $78 \%$ of the nationwide fleet of road vehicles, and serve as the basis of this simulation.

A household travel survey conducted between 2007 to 2010 shows that personal vehicles are in use an average of $3.3 \%$ of the time over a year, and that the average distance driven per day is $28 \mathrm{~km}$ in three trips [25]. Given typical present-day electric vehicles with a range of $150-160 \mathrm{~km}$ and battery capacity of 16-24 kWh (Mitsubishi MiEV, Nissan Leaf), up to $350 \mathrm{~km}$ and $56 \mathrm{kWh}$ (Tesla Roadster), there is significant potential for grid storage. For example, with a fleet of 1 million electric vehicles (40\% of the total light vehicle fleet), each with a $50 \mathrm{kWh}$ battery, and assuming that only $3.3 \%$ on average are in use at any time, $48 \mathrm{GWh}$ of storage would be available. In New Zealand, this is sufficient to supply peak load for three hours. Of course, such a simplistic analysis ignores the effects of vehicles arriving at and departing from the grid during this period; effects that the simulation does cover.

During 2010, 150,000 light passenger vehicles entered the fleet, while 110,000 were retired. At this rate, it would 
take about 17 years to replace the entire fleet. However, the Ministry of Transport notes that the replacement rate over the last three years has dropped significantly from previous years, which is likely to result in a higher replacement rate over the next five years because of vehicles reaching the end of their useful lives [24]. Partly because of the relatively high cost of new vehicles, the average private light vehicle age in New Zealand, 12.8 years, and terminal odometer reading of $195,000 \mathrm{~km}$, are higher than many other developed countries.

Present adoption rates for electric vehicles in New Zealand (largely plug-in hybrid, PHEV) are low, but one recent study which was concerned with the impact of electric vehicle charging on electricity requirements, suggests takeup rates of between $50 \%$ and $80 \%$ of new private light vehicles entering the fleet by 2040 [26]. At this estimate, and assuming linear growth in the proportion of electric vehicles from $0 \%$ in 2010 to $50 \%$ in 2040 , we calculate a total proportion of electric vehicles in the fleet of 1 million by 2040 , or 1.6 million given an $80 \%$ take-up rate, of a total fleet size of 4 million.

\section{SIMULATION OVERVIEW}

Although the simulation provides for individual vehicle representation in its present form, it deals only with system wide aspects of the interactions between the vehicles and the electricity network. It is intended that as the work progresses, the simulation will be expanded to include localized effects such as transmission and distribution flows, since a real system needs to take into account transmission line and transformer constraints.

Fig. 2 provides a general overview of the simulation. On the left are generation sources that only feed power into the grid, while the bulk load on the right only consumes power. The vehicle fleet allows energy flows in both directions.

Each of the components of this model, and the data on which they are based, are described in the following sections.

The simulated generation consists of a fixed base level, plus variable wind and peak generation. This is similar to the approach used by Inage [10], but differs in that wind generation is based on real wind speed data rather than a statistical model. The only generation source that varies in response to load is the peak generation. The EV fleet is primarily responsible for maintaining balance between generation and load, while peak generation fills in any shortfall that cannot be met by the fleet.

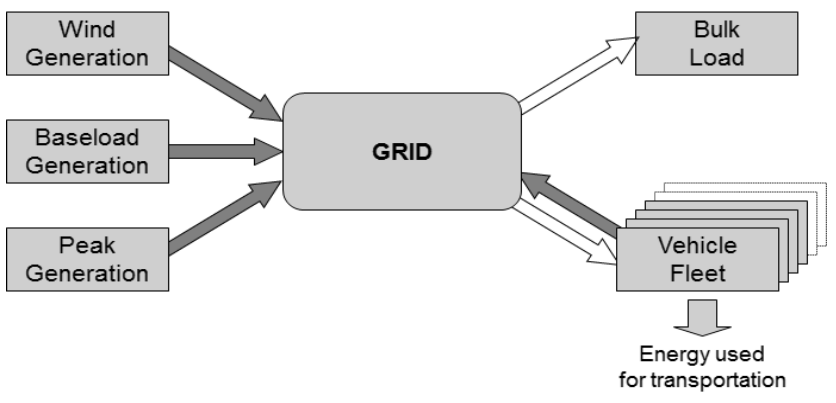

Figure 2. Block diagram of the simulation structure.

\section{A. Base Generation}

For the purposes of this simulation, base generation has a constant output such that base generation plus average annual wind generation is equal to the average load, including the additional requirements of the electric vehicle fleet (i.e. energy used for transportation). In reality, there is a variety of base generation such as hydro, geothermal and thermal, each with different characteristics in terms of responsiveness to changes in load and of energy storage, but for this initial evaluative stage of the simulation, such complexities have been ignored. Also ignored are seasonal variations in base generation to match demand. The ratio of wind capacity to base generation is set to test different levels of wind penetration.

\section{B. Wind Generation}

To model the combined output of current and proposed New Zealand wind farms, the simulation uses a synthetic wind speed data set described by Turner et al. [27]. This consists of time series wind speed data for 15 current and potential wind farm locations at 10-minute intervals over several years. However, for commercial reasons, the wind speeds for each site are disguised by either normalizing, or not revealing mast height or co-ordinates. This does not cause a problem for our simulation, since we are more interested in the variation in wind speed than we are in an absolute value. According to Bull [22], the relationship between wind speed and power output is approximately linear once factors such as turbine characteristics and farm layout are taken into account.

To use this dataset in the model, all 15 sites are first averaged for each 10-minute interval to create one large wind farm, using only data for the year 2007, since this is the most recent complete year in the dataset. In order to map wind speed to a power output, the wind speed at which the wind farm produces its full output must first be calculated. According to the New Zealand Wind Energy Association [20], New Zealand wind farms have an average capacity factor of $40 \%$. Since the average synthetic wind speed for 2007 was $9.44 \mathrm{~ms}^{-1}$, the nameplate power output will be achieved at wind speeds of $23 \mathrm{~ms}^{-1}$. Wind turbines can continue to generate maximum power above this speed. The formula to map wind speed to power output is therefore:

$$
W_{t}=\min \left(\frac{\text { wind speed }[t]}{\text { optimal speed }}, 1.0\right) \times \text { nameplateCapacity }
$$

\section{Peak Generation}

Peak generation fills in the shortfall between generation and bulk load. These generators are highly responsive and dispatchable, but commonly burn fossil fuels and are less efficient than base generators [6]. For this reason, one of the simulation goals is to minimize the use of peak generation by smoothing out fluctuations in both wind generation and electricity consumption using the EV fleet. The peak generation requirement is therefore an output of the simulation rather than a model within it. 


\section{Bulk Load}

It is essential to model electricity consumption in order to see the interactions between "normal" electricity consumption and that introduced by an electric vehicle fleet that supports bi-directional power flows. This is achieved by "playing back" zone load data for the year 2011, provided by Transpower, New Zealand's transmission network operator [28].

The dataset contains both real and reactive power for the main load centers in New Zealand at five-minute intervals. At this stage, the simulation utilizes only the aggregate real power for the whole country.

\section{E. Simulation of the vehicle fleet}

There are two main aspects to the vehicle model in the simulation; (i) the electrical model, which makes charging/discharging decisions when a vehicle is connected to the grid, and (ii), the vehicle behavior model that determines the timing and energy use of trips made by individual vehicles.

These models utilize, and contribute to, the basic parameters that each vehicle uses during the simulation. Table 1 outlines the static parameters, which remain fixed throughout the simulation, while table 2 outlines the dynamic parameters, which represent the state of the vehicle during the simulation.

\section{1) Electrical Model}

When connected to the grid, each EV must decide whether it should be charging, discharging (supplying energy to the grid), or neither. Each vehicle makes this decision by itself at each tick of the simulation, modeling a concept referred to as "decentralized smart charging" [14].

Naive solutions could simply charge at the maximum rate until the battery is full, or charge at the minimum rate necessary to meet the requirements of the next trip, but these would not realize the potential benefits of V2G. Worse, the charging behavior of all EVs is likely to coincide with not only other EVs, but also with traditional electricity demand, and therefore amplify overall peaks. These, and other charging models, are characterized in more detail later.

TABLE I. STATIC PARAMETERS IN THE VEHICLE MODEL

$\begin{array}{lll}\text { Parameter } & \text { Value } & \text { Unit } \\ \text { Battery capacity } & 50 & \mathrm{kWh} \\ \text { Minimum state of charge } & 1 & \mathrm{kWh} \\ \text { Maximum charge rate } & 5 & \mathrm{~kW} \\ \text { Maximum discharge rate } & 5 & \mathrm{~kW} \\ \text { Battery-to-wheel efficiency } & 110 & \mathrm{kWh} / \mathrm{km}\end{array}$

TABLE II. DYNAMIC VARIABLES IN THE VEHICLE MODEL

\section{Parameter}

Present state of charge

Next trip departure

Next trip distance

Next trip average speed

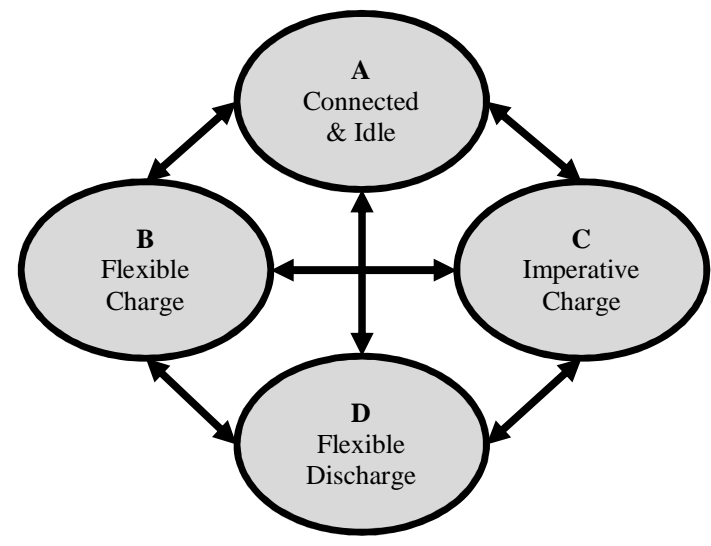

Figure 3. Finite state machine (FSM) to execute EV charging decisions.

TABLE III. SYMBOLS USED IN THE VEHICLE CHARGING FSM

$F \quad$ The vehicle is fully charged

$N \quad$ The grid has a shortage of generation

$P \quad$ The vehicle is able to supply energy to the grid

State $A$ Idle, when: $\bar{P} \cdot F+F \cdot \bar{N}$

State $B \quad$ Flexible charging, when: $P \cdot \bar{F} \cdot \bar{N}$

State $C$ Imperative charging, when: $\bar{P} \cdot \bar{F}$

State $D$ Flexible discharging, when: P.N

TABLE IV. SYMBOLS USED TO CALCULATE EV CHARGE POWER

$B \quad$ Base generation (W)

Eav Energy available in the EV fleet $(\mathrm{J})$

$\mathrm{Er} \quad$ Energy required to fully charge the EV fleet $(J)$

L Bulk load $(W)$

Lev Imperative EV charging load $(W)$

$q \quad$ Current battery charge of an $E V(J)$

Q1 Battery charge required by time $T 1(\mathrm{~J})$

Qmax Maximum battery capacity of an $E V(J)$

Qmin Minimum allowable EV battery charge $(J)$

Pmax Maximum charge power to an EV $(W)$

$S \quad$ Surplus generation $(W)$

st $\quad$ Current simulation date and time

$t \quad$ Simulation tick number

T1 Time of next departure of an EV

$W \quad$ Wind generation output $(W)$

$$
\begin{gathered}
S_{t}=B_{t}+W_{t}-\left(L_{t}+\operatorname{Lev}_{t}\right) \\
E r_{t}=\sum_{i=1}^{n} \operatorname{Qmax}_{i}-q_{i}
\end{gathered}
$$

$\operatorname{Eav}_{t}=\sum_{i=1}^{n} q_{i}-\max \left(Q \min _{i}, Q 1_{i}-P \max \times\left(T 1_{i}-s t\right)\right)$

The co-operative model proposed for the simulation makes smarter decisions based on internal information, such as state of charge, battery capacity, the requirements of the next trip, and external information including the current surplus/deficit of generation on the grid, and aggregate battery state of other grid-connected EVs.

In a real system, knowing the state of charge and battery capacity is relatively straightforward, while Kempton and 
Tomic [4] mention possible ways of specifying or learning patterns of use to estimate the requirements of the next trip. External information could be collected using existing communication networks, or it may be possible to infer this by analyzing the changes in grid frequency that result from imbalances between generation and load.

The models described below refer to the finite state machine description of Fig. 3, and the explanatory notes and formulae of Tables III and IV.

To calculate an appropriate charge rate, a vehicle needs external information; current surplus generation (2), the energy required to fully charge all connected EVs (3) and the current level of energy in the connected EVs that is available for grid management purposes (4).

The simulation calculates these values on each tick, but these values are not available to vehicles until the following tick. This is consistent with reality, as it is not possible to know the state of the grid at the time of measurement, but only shortly afterwards. This is especially true when grid state is inferred using changes in grid frequency.

In state $\mathrm{A}$, charge power is zero, while in state $\mathrm{C}$, charge power is simply the maximum rate possible for the vehicle. Charge power for states B and D require further calculation.

For state B, vehicles that are near their full capacity are charged at a lower rate than those that are near empty, according to (5).

$$
\operatorname{Pin}=S_{t-1} \times \frac{Q \max -q}{E r_{t-1}}
$$

For state D, vehicles that are near their full capacity are discharged at a higher rate than those that are near empty, according to (6).

$$
\text { Pout }=S_{t-1} \times \frac{q-\max (Q \min , Q 1-P \max \times(T 1-s t))}{E a v_{t-1}}
$$

The desired effect of these equations is to balance the charge between each EV, while attempting to maintain the balance between generation and load at all times.

In order to compare the strengths and weaknesses of this charging strategy, several charging strategies have been evaluated:

- Co-op: the co-operative strategy described earlier in this paper.

- Greedy: an EV will charge at its maximum rate until fully charged. If not controlled, EV charging will take this form.

- Lazy: a "just in time" charging method. No charging will be done until as close as possible to the time of departure. At that point, the EV will charge at its maximum rate.

- Lazy+: similar to Lazy, but will charge in the interim if there is excess generation.

- Slow: an EV will charge constantly at the minimum rate required to complete the next trip.

2) Vehicle Behaviour Model

An important part of the simulation is to accurately model the times of day vehicles are used, how far they travel and the amount of time they spend on the road, in order to track both their energy requirements, and their availability for grid management purposes. The New Zealand Household Travel Survey [25] invited people from 4600 households each year to record all of their travel over a two-day period. The results of that survey have been used to generate trips in the simulation.

Each vehicle in the simulation knows its next trip, which consists of a time of departure, distance, and average speed. When a vehicle returns from a trip, it generates its next trip, and stays connected to the grid until departure.

To generate the next trip's time of departure, the cumulative distribution function of Fig. 4 is sampled 22 times, so that trips are more likely to occur at the most popular times of day. The sample that is immediately after the current simulation time will be the time of next departure, wrapping back to the start of the next week where necessary. The distribution is sampled 22 times, since this is, on average, the number of trips made each week [25].

Once the departure time is calculated, an average distance is taken from the distribution shown in Fig. 5. Next, a random sample is taken from a distribution of the shape shown in Fig. 6, which is the distribution of trip distance per day, but normalized to have a mean of one. The average trip distance from Fig. 5 is then multiplied by this value and becomes the distance of the next trip.

Finally, the average speed of the trip is simply chosen to be $36 \mathrm{~km} / \mathrm{h}$, a figure indicated by the Household Travel Survey [25].

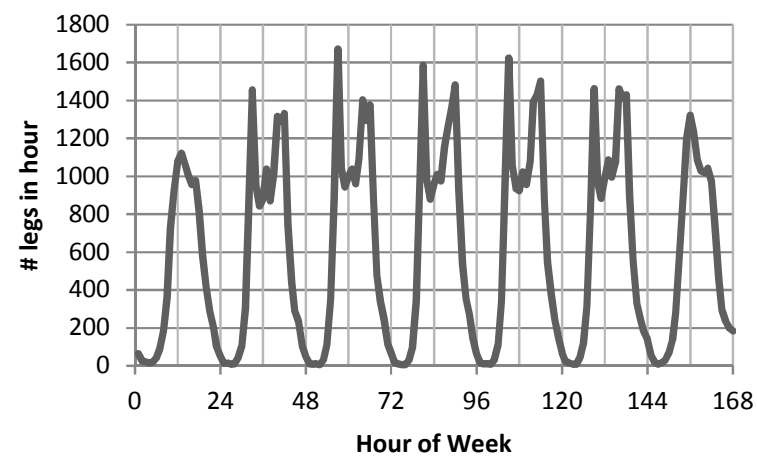

Figure 4. Trip legs by hour of week [25].

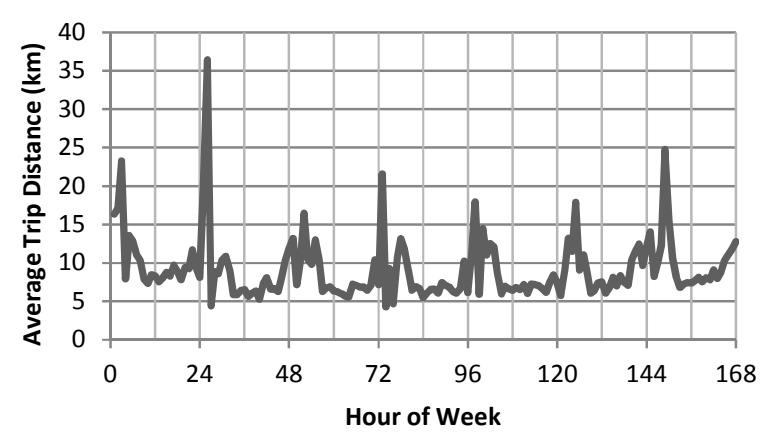

Figure 5. Trip distance by hour of week [25]. 


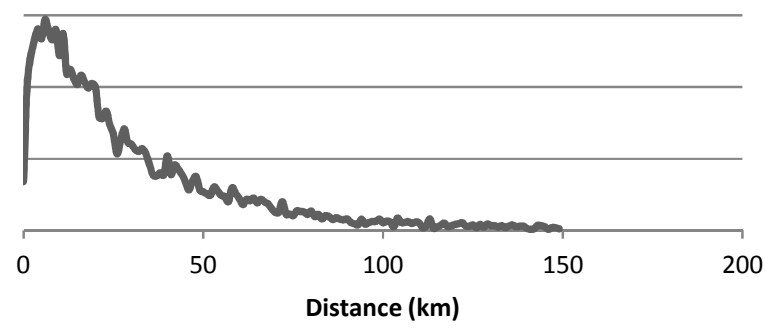

Figure 6. Distribution of daily travel distance per vehicle [25].

Immediately after generating the next trip, a check is performed to ensure that the trip is possible. It may not be, either because the distance is further than the range of the vehicle, or it is scheduled to depart before the vehicle can be bought up to a sufficient charge. In either case, the failure is recorded and a new trip generated to replace it.

\section{RUNNING THE SIMULATION}

The simulation works on a tick-by-tick basis. At the beginning of the simulation, the state is often unsettled - the entire EV fleet having empty batteries, for example. This is addressed by running the simulation for a day before the intended start time, allowing state to settle down - a similar approach used in [16].

The duration of a single tick can be changed dynamically. Smaller tick duration means greater output resolution and accuracy, at the expense of slower execution.

A five-minute tick interval has been chosen, as our finest-resolution dataset (bulk electricity load) is of this duration. Not all models are derived from data sources with this resolution; in these cases, linear interpolation between data points is used.

The process that is executed for each tick can be described as follows:

1. For each vehicle that has departed since the last tick, disconnect from the grid.

2. For each vehicle that has returned from a trip since last tick, update battery state, reconnect to the grid and generate the next trip.

3. Look up values for wind generation, base generation, and bulk load from their respective models.

4. For each connected EV, make the charging decision described previously.

5. Add the power input/output of all entities connected to the grid to calculate overall power imbalance.

6. Record the current state of the network, including generation breakdown, peak generation needed to maintain balance between generation and load, and the state of the EV fleet.

7. Calculate values for (2), (3) and (4) for use at the next tick, as described in the previous section.

In a real electricity network, generation must match load at all times; an output of the simulation at each tick is the amount of additional generation required to keep this balance. This could well be negative, implying that some energy must be spilled, by leaving potential energy (e.g. wind) unutilized.

\section{RESULTS}

The results presented here were generated by running the simulation for a one-year period, with wind penetrations of $10 \%, 20 \%, 30 \%, 40 \%$ and $50 \%$, and with 1 million EVs. The generation model consists of only wind and a fixed base generation level, which were set to sum to the average demand over the year (4.6 GW average electricity load, plus an additional $130 \mathrm{~W}$ for each EV). The proportion of wind to base generation is set according to the wind penetration under test.

\section{A. Peak Generation and Spillage}

For the purposes of discussion, "peak generation" refers to the generation needed, in addition to wind generation, base generation and output of the EV fleet, to maintain balance between generation and demand. Peak generation is only used when the EV fleet cannot supply the necessary power. Similarly, "spillage" refers to electricity that could be generated, but must necessarily be wasted because there is neither demand nor storage available to absorb it. In effect, a non-zero peak generation requirement results from the failure of the EV fleet to balance generation and load.

By using the storage of a fleet of 1 million EVs to cover generation shortages, it is possible to substantially decrease the energy required from peak generation sources. Fig. 7 shows that the co-op charging strategy performs much better than all other charging strategies, including the case where no EVs are present. A similar situation exists for energy spillage, shown in Fig. 8. Lazy+ reduces energy spillage by absorbing the energy when it is available, and does not require as much peak generation later - functionally equivalent to storage, but more efficient because energy is not converted back to AC from the batteries.

Note that in the "No EVs" case, simulated generation (base + wind) is lower than in cases that include EVs, since the provision of $130 \mathrm{~W}$ average power per EV is not required.

\section{$\square 10 \%$ Wind $\square 20 \%$ Wind $\square 30 \%$ Wind $\square 40 \%$ Wind $\square 50 \%$ Wind}

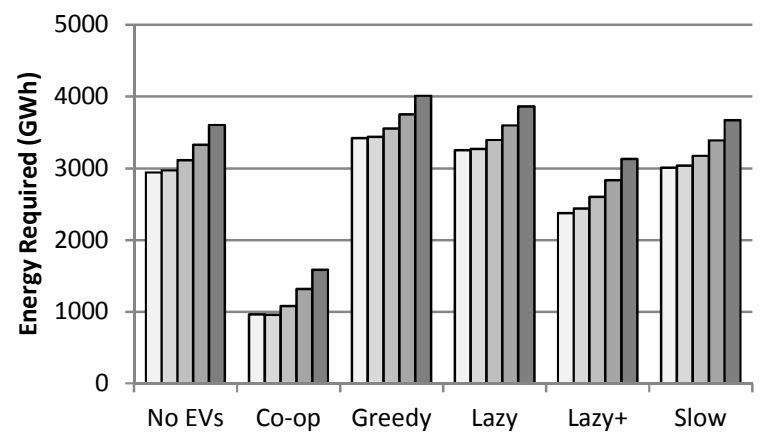

Figure 7. Annual peak generation required. 
$\square 10 \%$ Wind $\square 20 \%$ Wind $\square 30 \%$ Wind $\square 40 \%$ Wind $\square 50 \%$ Wind

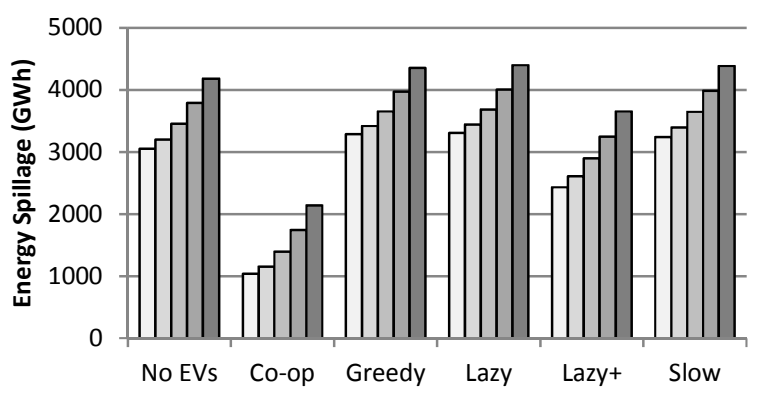

Figure 8. Annual energy spillage by wind penetration.

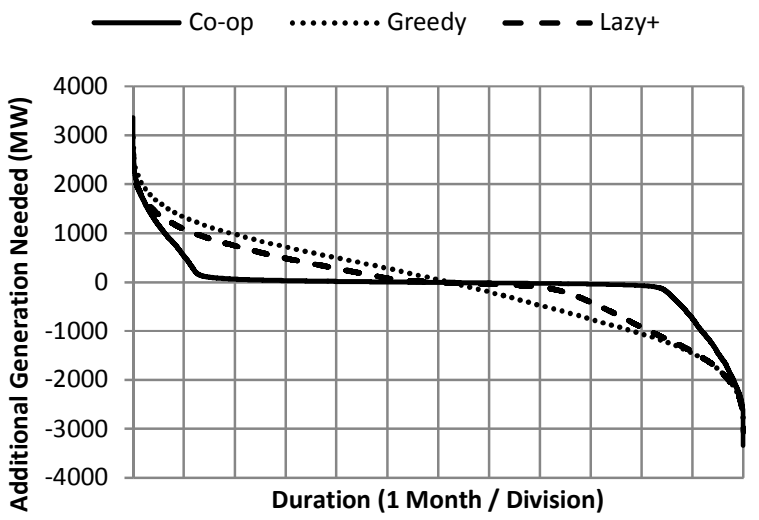

Figure 9. Annual peak generation load-duration curve, $30 \%$ wind.

The load-duration curve for the peak generation required over the year (Fig. 9) shows that co-op maintains balance between generation and demand for about $75 \%$ of the year, a significant improvement over greedy and lazy+.

The highest use of peak generation for co-op was as high as for other charging strategies. These peaks occur during periods of high bulk demand and low wind generation, where $\mathrm{V} 2 \mathrm{G}$ makes up a high proportion of input to the grid. If such situations are prolonged, the EV fleet will be drained of energy, and a sudden input of peak generation will be required to cover not only the bulk demand, but also the imperative transport requirements of the EV fleet. It should be possible to predict this situation hours, if not days, before they occur. Peak generation could then be bought online earlier to spread the load over a longer period.

\section{B. Electricity Demand}

In terms of overall electricity demand, the introduction of an EV fleet using the greedy charging strategy increases load during higher demand periods, while utilizing very little offpeak capacity (Fig. 10). Using the co-op charging strategy increases the electricity load primarily during off-peak times, while contributing energy back to the grid during higher demand (Fig. 10). This behavior is a desired side effect of the strategy in terms of infrastructure requirements; however, the primary aim is to match supply and demand.

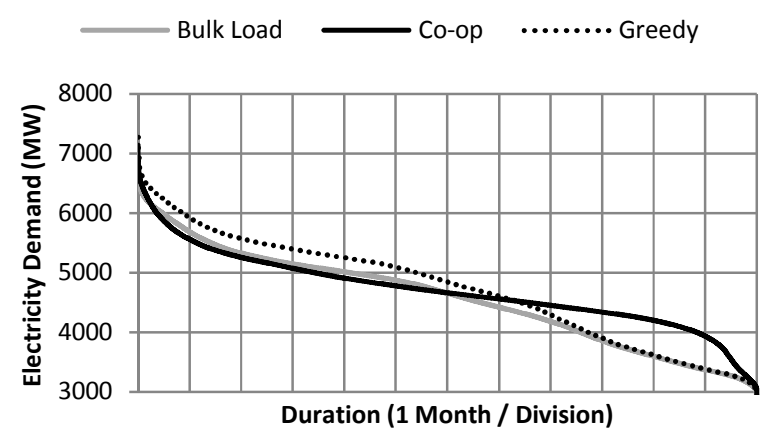

Figure 10. Annual demand load-duration curve, $30 \%$ wind.

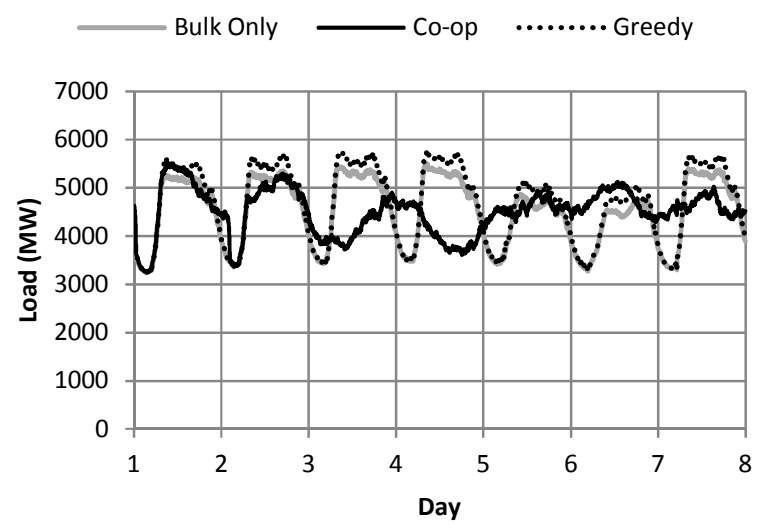

Figure 11. Load during the first week of February 2011, 30\% wind.

Fig. 11 shows the demand curve during the first week of February (with the weekend occurring on the $5^{\text {th }}$ and $6^{\text {th }}$ ). Again, this shows how the greedy strategy contributes to peaks, and does not effectively utilize off-peak capacity, while the co-op strategy effectively follows the variable output from the wind generation model.

There are two cases of energy spillage occurring during the period shown in Fig. 11 using the co-op strategy. This occurs during the early hours of the first and second day of the month. Overall load drops to the original bulk demand level when the EV fleet becomes fully charged, and resumes following wind generation once demand rises later in the day.

\section{Aggregate Battery Charge}

An EV fleet of one million vehicles, each with a battery capacity of $50 \mathrm{kWh}$, has a storage capacity of $50 \mathrm{GWh}$. Over the year, the grid-connected storage capacity averages 48 GWh with a minimum of $46 \mathrm{GWh}$, while actual charge level averages $25 \mathrm{GWh}$, of which $24 \mathrm{GWh}$ is available for grid management purposes. This is sufficient to completely provide New Zealand's average electricity demand for five hours in the complete absence of generation. Of course, real values will be lower, since the simulation currently works on the assumption that a vehicle is connected to the grid at all times that it is not being driven. 


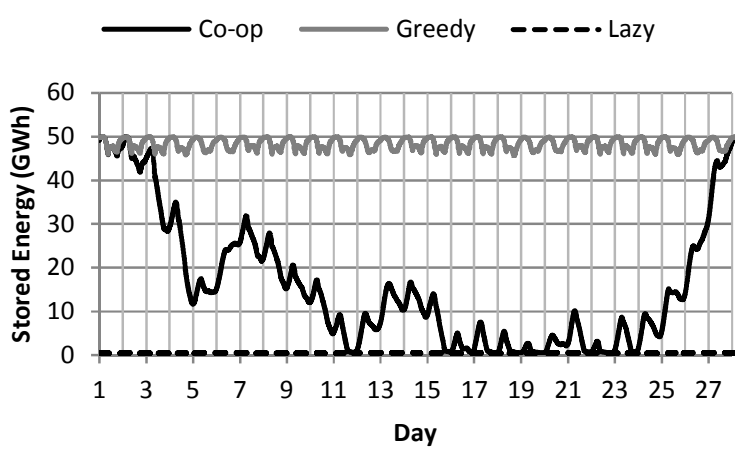

Figure 12. Aggregate EV battery charge during February 2011,30\% wind.

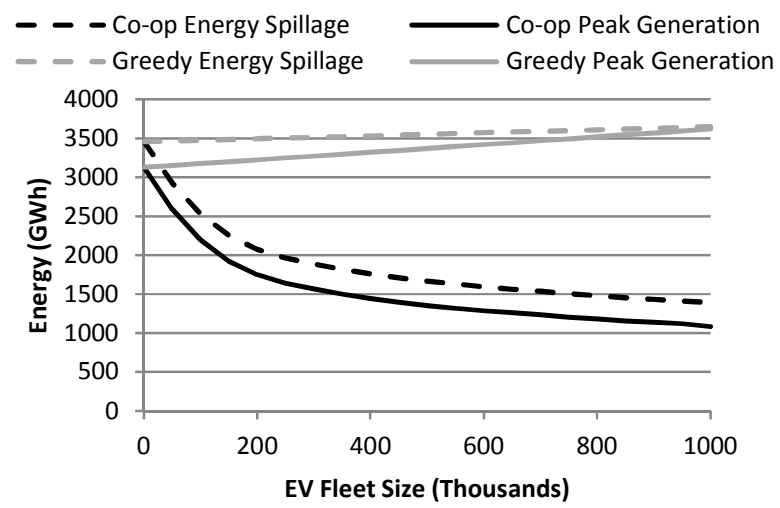

Figure 13. Peak Generation and Spillage by Fleet Size, 1 year, 30\% wind

Fig. 12 shows the aggregate energy stored in the EV fleet's batteries over the month of February. Not surprisingly, the greedy strategy maintains batteries near full capacity. The daily variation shown is primarily a result of vehicles being connected and disconnected from the grid. The lazy charging strategy tends to maintain batteries near empty, since they will be charged as late as possible before departing on the next trip.

The difference in charge between these two strategies is available for grid management purposes, with the maximum charge bounded by the levels shown under the greedy strategy, and the minimum bounded by the lazy strategy.

The co-op strategy most commonly charges overnight, which is evident in Fig. 12, but may charge at any time when generation exceeds load. When the EV fleet's charge reaches the maximum, any excess generation is spilt. Conversely, when $\mathrm{EV}$ charge is at a minimum, further input is required from peak generation to maintain balance.

The problem that the co-op charging strategy has regarding high peak demands could potentially be addressed by setting a threshold when peak generation is brought online, for example, when EV capacity drops below 10 $\mathrm{GWh}$, rather than only taking action once the fleet is empty. Energy shortages would therefore be spread over a longer period, reducing demands on both generation and transmission infrastructure. The timing of bringing peak generation online is not critical, as the EV fleet provides significant flexibility.

\section{Unchargable Trips}

A top priority of any EV charging strategy should be to ensure that all trips can be made when required. Several of the strategies have the drawback of providing only for the next trip. In particular, the lazy and slow strategies attempt to provide only the minimum energy necessary for the next trip. If, for example, a 10-minute trip was followed shortly afterwards by a 2-hour trip, it would not be possible to charge the vehicle between trips because of time and power constraints. Using the lazy strategy, 5\% of all trips could not be charged in time, compared to $1 \%$ when using the Co-op strategy. Since the greedy strategy maintains batteries near full capacity, it does not suffer from this effect.

\section{E. EV Fleet Size}

Fig. 13 shows how the energy balance over the simulated year is affected by fleet size. The reductions of energy required from peak generation, and in energy spillage, exhibit a law of diminishing returns. An EV fleet size of one million vehicles delivers only about $35 \%$ savings over a fleet size of 200,000 . This suggests that only a part of the overall fleet needs to use the co-op charging strategy to realize the benefits, and the strategy will be useful even if $\mathrm{EV}$ uptake is lower than expected. In fleet sizes of less than 200,000, the primary limitation of the load-matching ability of the fleet is the power capacity of the vehicle to grid connection, while fleets greater than this size are primarily constrained by the energy storage capacity of the batteries. Other important factors, such as battery life implications and the charging locations within the electricity network are not taken into account. When allowing for these factors, the benefits of high participation in the co-op strategy could be enhanced.

When using the greedy charging strategy, both the energy required from peak generation sources and the energy spillage increase as the fleet size increases, the opposite effect seen when using the co-op strategy.

\section{DISCUSSION AND FUTURE WORK}

While the results show promise regarding the potential of $\mathrm{V} 2 \mathrm{G}$ to balance intermittent generation in New Zealand, there remain many unsolved challenges. The simulation is an ideal tool for investigating many of these challenges, and in future work will be expanded to explore the operation of a future electricity grid in much more detail.

Of particular interest are the local and regional effects of the integration of intermittent generation, including distributed generation, the widespread adoption of EVs into distribution networks, and how the challenges of accommodating these new energy flows could be addressed.

In addition to wind generation, other renewable and intermittent energy sources may become more common, such as photovoltaic and tidal. The simulation could include models of these sources to explore the implications of the various output patterns. In addition to exploiting the storage 
capacity of an EV fleet, the simulation could further evaluate the load matching potential from other mechanisms such as domestic electricity consumption management. Accurate modeling of existing hydroelectric systems is also important, because of the variable nature of inflows and the storage potential existing within those systems. The simulation could also be used to evaluate the requirements for other forms of energy storage, such as long-term pumped hydro [21].

This paper looks only at a homogenous EV fleet, but this is not expected to be realistic. It would be useful to evaluate a mixture of vehicle technologies, such as hybrid or fuel cell vehicles, all with different capabilities, with detailed vehicle models that include battery life considerations. In addition to improving upon the co-operative charging strategy, the simulation could include a mixture of other strategies.

Finally, if V2G is to become widespread, there must be a suitable economic model for its adoption. The simulation is well placed to incorporate economic considerations, such as the value of V2G to vehicle owners and grid operators, the battery life implications, and other factors that could influence the overall success of the approach.

\section{CONCLUSION}

This paper describes a simulation framework that can be used to model the behavior of an electricity network following the introduction of an electric vehicle fleet and high wind generation penetration. It shows how the distributed battery capacity of an EV fleet can be utilized to allow greater proportions of intermittent renewable generation in an electricity grid without relying heavily on alternative peak generation.

Models for various parts of New Zealand's electricity network have been developed, including bulk load, wind generation and vehicle use, using real and projected data.

A co-operative vehicle charging strategy has been described, where each vehicle makes charging decisions independently, while taking into account external factors such as the current surplus/deficit of generation, charge state of other vehicles, as well as internal factors such as charge state and the details of the next trip.

Finally, the results from an initial simulation run of the grid over a one-year period have been presented, that demonstrate the potential benefits of using V2G technology and some of the capabilities of the simulation itself. The initial trial shows that with a relatively simple charging strategy, and access to information about the current state of the grid, the proportion of wind generation can be increased beyond received norms without requiring significant energy from peak generation sources.

\section{REFERENCES}

[1] W. Kempton and S. E. Letendre, "Electric vehicles as a new power source for electric utilities," Transportation Research Part D: Transport and Environment, vol. 2, no. 3, pp. 157-175, 1997.

[2] D. J. MacKay, Sustainable Energy - without the hot air. UIT, Cambridge, UK, 2009.

[3] A. Franco and P. Salza, "Strategies for optimal penetration of intermittent renewables in complex energy systems based on techno-operational objectives," Renewable Energy, vol. 36, no. 2, pp. 743-753, 2011.

[4] W. Kempton and J. Tomić, "Vehicle-to-grid power implementation: From stabilizing the grid to supporting largescale renewable energy," Journal of Power Sources, vol. 144, no. 1, pp. 280-294, Jun. 2005.

[5] G. N. Bathurst and G. Strbac, "Value of combining energy storage and wind in short-term energy and balancing markets," Electric Power Systems Research, vol. 67, no. 1, pp. 1-8, Oct. 2003.

[6] M. Black and G. Strbac, "Value of storage in providing balancing services for electricity generation systems with high wind penetration," Journal of Power Sources, vol. 162, no. 2, pp. 949 953, 2006.

[7] M. B. Blarke and H. Lund, "The effectiveness of storage and relocation options in renewable energy systems," Renewable Energy, vol. 33, no. 7, pp. 1499-1507, Jul. 2008

[8] D. Connolly, "The Integration of Fluctuating Renewable Energy Using Energy Storage," PhD Thesis, University of Limerick, 2010.

[9] D. Connolly, “A Review of Energy Storage Technologies: For the integration of fluctuating renewable energy (Version 4)," Research Report, University of Limerick, 2010.

[10] S.-I. Inage, "Modelling Load Shifting Using Electric Vehicles in a Smart Grid Environment," OECD/IEA Working Paper, No. 2010/07, 2010.

[11] W. Short and P. Denholm, "A preliminary assessment of plug-in hybrid electric vehicles on wind energy markets," NREL Report no. TP-620-39729, 2006

[12] G. Haines, A. McGordon, P. Jennings, and N. Butcher, "The Simulation of Vehicle-to-Home Systems - Using Electric Vehicle Battery Storage to Smooth Domestic Electricity Demand," in Ecological Vehicles and Renewable Energies Conference (EVER), 2009.

[13] Y. Ma, A. Cruden, M. Iet, and D. Infield, "A Matlab Simulator for Electric Drive Vehicle to Grid Implementation," Power, vol. 4, pp. 1097-1101, 2010.

[14] R. A. Waraich, M. D. Galus, C. Dobler, M. Balmer, G. Andersson, and K. W. Axhausen, "Plug-in Hybrid Electric Vehicles and Smart Grid: Investigations Based on a MicroSimulation," ETH Zurich, Switzerland, 2009.

[15] W. Kempton and J. Tomic, "Vehicle-to-grid power fundamentals: Calculating capacity and net revenue," Journal of Power Sources, vol. 144, no. 1, pp. 268-279, 2005.

[16] D. Dallinger, D. Krampe, and M. Wietschel, "Vehicle-to-Grid Regulation Reserves Based on a Dynamic Simulation of Mobility Behavior," IEEE Transactions on Smart Grid, vol. 2, no. 2, pp. 302-313, Jun. 2011.

[17] H. Lund and W. Kempton, "Integration of renewable energy into the transport and electricity sectors through V2G," Energy Policy, vol. 36, no. 9, pp. 3578-3587, 2008.

[18] Ministry of Economic Development, "New Zealand Energy Data File,” Report, ISSN 1177-6684, 2011.

[19] Ministry of Economic Development, New Zealand Energy Strategy - Developing our energy potential. Report, ISBN 978-0478-35894-0, 2011.

[20] New Zealand Wind Energy Association, "Wind Turbine Technology," 2011. [Online]. Available:

http://windenergy.org.nz/documents/factsheets/technology.pdf [Accessed: 27-Jan-2012].

[21] W. E. Bardsley, "Note on the pumped storage potential of the Onslow-Manorburn depression, New Zealand," Journal of Hydrology (NZ), vol. 44, no. 2, pp. 131-135, 2005.

[22] B. Bull, "Correlation between wind generation output and hydro inflows," Report prepared for the Electricity Commission, 2010. Statistics New Zealand, "National Population Estimates: September 2011 quarter," 2011. [Online]. Available: http://www.stats.govt.nz/browse_for_stats/population/estimates_ and_projections/NationalPopulationEstimates_HOTPSep11qtr.as px. [Accessed: 27-Jan-2012] 
[24] Ministry of Transport, "The New Zealand Vehicle Fleet," Statistical Report, ISBN 978-0-478-07228-0, 2011.

[25] Ministry of Transport, "New Zealand Household Travel Survey 2007 - 2010: Driver travel," Fact Sheet, 2011.

[26] New Zealand Centre for Advanced Engineering (CAENZ),

"Electric Vehicles - Impacts on New Zealand's Electricity System,” Technical Report, 2010.
[27] R. Turner et al., "Multi-year ten-minute synthetic wind speed time-series for 15 actual or proposed New Zealand wind farms," Client Report, NIWA, 2009.

[28] Transpower, "Zone Load Graphs." [Online]. Available: http://systemoperator.co.nz/n1944,download=true.html. 